\title{
Cardiac CT Image Enhancement Method Based on Prior Knowledge
}

\author{
XU Zihang ${ }^{1, a}$ JIANG Rui ${ }^{2, b}$ \\ ${ }^{1}$ Faculty of Information Technology, Macau University of Science and Technology, Macau, China \\ ${ }^{2}$ Faculty of Information Technology, Macau University of Science and Technology, Macau, China \\ xzh_58@163.com¹458952792@qq.com ${ }^{\mathrm{b}}$
}

Keywords: CT, image enhancement, cardiac positioning, prior knowledge

\begin{abstract}
Despite the application of contrast enhancement to image enhancement in medical image processing and in spite of a good enhancement effect achieved, since the priori knowledge of the relevant application domain is left out, the medical images affected by noise have yet to be further enhanced. Priori knowledge-based contrast enhancement can well eliminate noise effect by extracting known part of mechanism from an object as priori knowledge and combining it with sample data to build a reliable sample model. This paper proposed a priori knowledge-based cardiac CT image block adaptive contrast enhancement algorithm, which can be used to process various characteristics of a cardiac CT image flexibly to general an ideal enhanced image.
\end{abstract}

\section{Introduction}

Image enhancement is very important in the field of image processing, and has been one of the research hotspots in this field for many years [1-2]. Image contrast enhancement usually exists as a preprocessing module of image processing system, of which the input is generally the original image which has low contrast and is difficult to meet the requirements of subsequent algorithms and the output is the enhanced result that the contrast is improved to meet the requirements of subsequent algorithms. Contrast enhancement algorithm is the process where a kind of brightness correction function is used to correct the original gray value [3-4]. The existing algorithms are subject to analysis and processing of the whole image, and the local information of the image is not well utilized. Therefore, we introduce a block processing technique, which makes use of the concept of local analysis and processing to divide the image into several sub-blocks for analysis and processing. Thus, the local information can be better used. Based on the prior knowledge, research and extensive test experiments of the contrast enhancement method are carried out. This paper proposes an image block-adaptive contrast enhancement algorithm based on prior knowledge.

\section{Priori Knowledge}

Priori knowledge refers to all available information except training data for a learning task, which covers a wide range. The two characters hardest to distinguish from each other in automatic character recognition are 0 and $\mathrm{O}$, because there is the shortest between-class distance between them, and recognition errors are generally caused by them. But according to priori knowledge, we may know that there can only be a specific character in some location. For instance, it is very possible that there is $\mathrm{O}$ rather than 0 among a string of letters a string of letters; on the contrary, it is very possible that there is 0 rather than $\mathrm{O}$ around an array of Arabic numerals. At present, the scholars in the artificial intelligence domain have introduced priori knowledge into SVM classification model for research in the following two major areas: 1) kernel function selection for specific background knowledge; 2) direct introduction of priori knowledge into SVM classification model, including direct introduction of priori knowledge attributes into training samples, followed by unified representation through SVM. The latter is adopted in the paper. 


\section{Feature extraction of image sub block}

An image contains abundant information, from which lots of characteristic parameters representing image characteristics can be extracted. An analysis of these characteristic parameters can help determine what enhancement function may be used to process the original image to achieve an ideal enhancement effect. The priori knowledge-based adaptive image enhancement algorithm is in itself a contrast enhancement algorithm based on this mechanism. The first problem needing solution for an adaptive algorithm is that such characteristic parameters must be extracted effectively from image content. The existing adaptive algorithms can hardly achieve a satisfactory processing effect since local information characteristics fail to be well utilized. This paper put forward a local analytical processing algorithm, which can achieve a more precise enhancement effect since it extracts characteristic parameters from local information for image enhancement.

For certain image blocks, the mean brightness $\mathrm{m}$ and contrast $\mathrm{c}$ of the blocks are extracted as characteristic parameters in this region:

$$
\begin{gathered}
\mathrm{m}=\sum_{l=1}^{l_{\max }}\left[\frac{l}{l_{\max }} \cdot p d f(l)\right](1) \\
\mathrm{c}=\sum_{l=1}^{l_{\max }}(1-m)^{2} \cdot p d f(l)(2)
\end{gathered}
$$

where, $1(1 \in[0,255])$ represents grey level, 1 max is the maximum of 1 , and pdf is the normalized histogram of block pixel gray.

c represents the contrast of the original image, and the lower the contrast ratio is, the higher the enhancement effect should be. In order to build an organic relationship between image contrast and the final enhancement function gain parameters, we introduced a contrast-related parameterized grey probability distribution function:

$$
\operatorname{pdf}^{r(b)}=p d f_{\max }^{f} \cdot\left(\frac{p d f\left(h-p d f_{\min }\right.}{p d f_{\max }-p d f_{\min }}\right)^{(1+\sqrt{c})}(3)
$$

where, pdf_max and pdf_min are the maximum and minimum of various elements in $1 \mathrm{D}$ vector $\mathrm{p}$ $\mathrm{d}$ f respectively, and pdf is a probability distribution function of normalized discrete gray value:

$$
\operatorname{pdf}(l)=\frac{\sum_{i=1}^{M} \sum_{j=1}^{N}[s(n)=l]}{M \times N}(4)
$$

where, $i$ and $j$ are the row and column coordinates of pixel points within the window respectively; $M$ and $N$ are the width and height of image blocks respectively, and that $i \in[1, M], j \in[1, N]$. In Formula (3), probability distribution functions of varying smoothness can be established if different values are assigned to parameter $\mathrm{c}$, and then different enhancement function curves can be established.

\section{Cardiac CT image blocking based on prior knowledge}

During the medical image processing, although the contrast enhancement algorithm of the whole image has been applied to the image enhancement, and achieved a good enhancement effect, the prior knowledge of the relevant application areas have not been taken into account. Enhancement effect on medical images which are affected by noise is required to be further improved. The image block-adaptive contrast enhancement algorithm based on prior knowledge can better solve the effect of noise. It extracts a known part of the object as the prior knowledge, which is then combined with the sample data to establish a reliable sample model. 

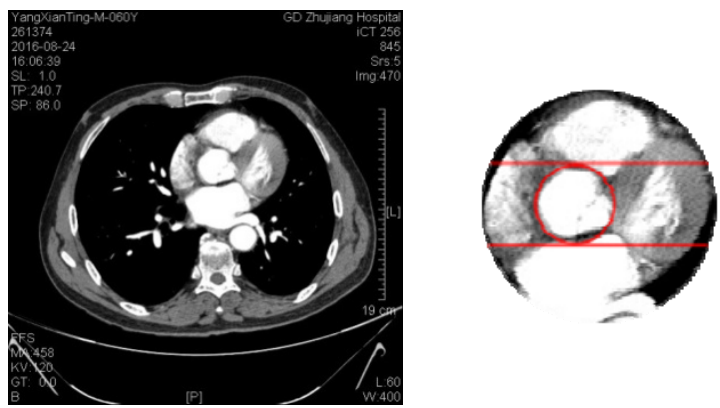

Fig.1 The sample data

Based on cardiac positioning, atria and ventricles required to be enhanced are determined. Then, concentric circles are used to divide the heart into different regions and carry out corresponding contrast enhancement.

\section{Block analysis and processing}

Complex images with mixed features are common in medical images. The gray histogram of image often has multi-peak characteristics. Thus, it is not easy to obtain ideal enhancement results of those complex image. However, if the image is divided into several sub-blocks, which are then separately analyzed and processed, mutual interference among different feature areas can be avoided when the whole image is being processed, further effectively extracting feature quantities and more accurately obtaining correction function. Then, after the image sub-blocks are successively processed and finally integrated into a complete enhanced image, the desired enhancement results can be obtained. The specific steps of the algorithm are as follows:

(1) Select the appropriate oval elliptic according to the image size and then circle the region of interest.

(2) Scan the entire image from left to right and top to bottom. According to the method described above, determine the center point position of the concentric circle and divide it into several blocks.

(3) Analyze and process each sub-block and store the processing results. Traverse the entire image to complete scanning.

\section{Summary}

We processed each cardiac CT image by histogram equalization (HE) [5], common contrast enhancement and the method proposed in this paper, and made a lateral correlation between the results. See Fig. 1 for the specific experimental results. To sum up, the algorithm proposed in this paper has strong adaptability to cardiac CT images, and its overall processing effect is significantly better than other algorithms. Due to the adoption of local analytical processing and a strongly adaptive priori knowledge mechanism, the algorithm proposed in this paper has certain adaptability and is superior to all other available algorithms. However, this research work is still in its infancy, and there are still many problems demanding solution. For instance, more use can be made of abundant image information to extract image characteristic parameters more precisely; a more effective adaptive generation mechanism can be adopted in the enhancement function to further improve the adaptability of the algorithm. These problems will be further researched in the future. 

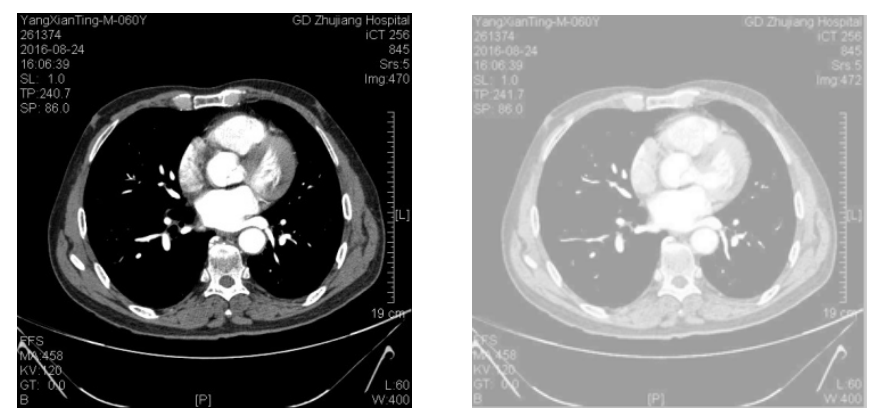

(a) original image

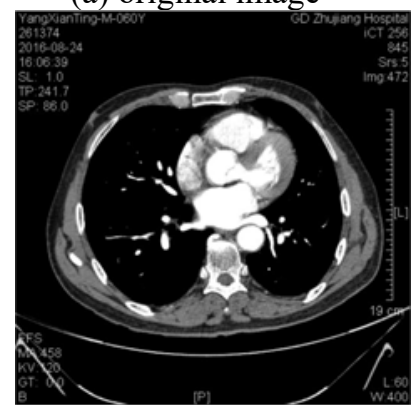

(c) Contrast enhancement (b) Histogram equalization

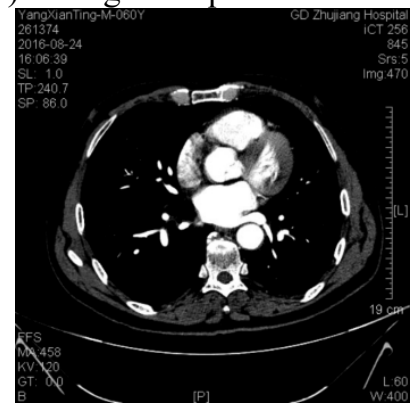

(d) Paper method

Fig. 2 Specific experimental results

\section{References}

[1] Smolic A, Mueller K, Merkle P, et al. Multi-View Video plusdepth (MVD) format for advanced 3D video system[C] // 23rdMeeting ISO/IEC JTCI/SC29/WGlland ITU-T SG16 Q. 6, JVT-w1OO. USA: San Jose,2007

[2] Maire M, Do M N. Joint Encoding of the Depth Image BasedRepresentations Using Shape-Adaptive Wavelets [C] // IEEEProceedings of the International Conference on Image Processing. 2008:1768-1771

[3] Wang M, Liu C, Zhang T, et al. Region of interest oriented fastmode decision for depth map coding in DIBR[C] // IEEE 7ternational Colloquium on Signal Processing and its Applications. 2011:177-180

[4] Christopoulus C» Askelof $\mathrm{J}$ » larsson M. Efficient regions of Interest coding Techniques in upcoming JPEG2000 still image coding standard[C] // Processings of International Conference onImage Processing(ICIP). 2000, 2 •41-44

[5] Yeganeh H, Ziaei Rezaie believes his new method for contrast enhancementBased on the histogram equalization [C] // journalsInternational conference on computer and communication

Engineering. Kuala lumpur, Malaysia: IEEE, 2001:256-26 